\title{
Thiomers: A Blessing to Evaluating Era of Pharmaceuticals
}

\author{
Muhammad Hanif, ${ }^{1}$ Muhammad Zaman, ${ }^{1,2}$ and Sundas Qureshi ${ }^{2}$ \\ ${ }^{1}$ Department of Pharmacy, Bahauddin Zakariya University, Multan, Pakistan \\ ${ }^{2}$ Faculty of Pharmacy, The University of Lahore, Pakistan
}

Correspondence should be addressed to Muhammad Zaman; m.zaman2157@gmail.com

Received 3 April 2015; Revised 4 June 2015; Accepted 10 June 2015

Academic Editor: Angel Concheiro

Copyright (C) 2015 Muhammad Hanif et al. This is an open access article distributed under the Creative Commons Attribution License, which permits unrestricted use, distribution, and reproduction in any medium, provided the original work is properly cited.

\begin{abstract}
Thiomers are the polymers modified for the mucoadhesive properties and other additive properties by incorporating thiol moieties in the backbone of the unmodified polymeric chain by substitution reactions or simple oxidation reactions. Drugs that are less soluble and permeable can be complexed with thiomers for their increased absorption through the mucosal membranes by increase in contact time and prolonged stay in body due to mucoadhesion. Immobilization of thiol group therefore increases the mucoadhesive properties of the modified polymer by $2-140$-folds. The prepared thiomers are characterized and made stable by different techniques. Thiomers also give the controlled delivery of the active pharmaceutical ingredients in the body. Different polymers that are modified by thiolation are chitosan, polyacrylic acid, sodium alginate, sodium carboxy methyl cellulose, guar gum, and so forth. Thiomeric formulations are a challenge to deliver drugs with low therapeutic compatibility. Micro- and nanopreparations containing thiomers can be prepared by different techniques such as covalent crosslinking, in situ gelation, radical emulsion polymerization, and emulsification. Nowadays thiomers have wide range of applications as a promising pharmaceutical excipient in the evaluating era of pharmaceutical technology.
\end{abstract}

\section{Introduction}

Bioadhesion is the phenomenon by which any molecule adheres to the biological membrane but when the adherence to the mucus is specified it is referred to as mucoadhesion [1]. Mucoadhesion is introduced in the pharmaceutical field for more than 40 years and it is of main importance in oral delivery systems, as drugs taken from oral route have various bioavailability issues $[2,3]$. Adhesion to the membranes is of importance as it prolongs the stay of the drug in the body subsequently increasing bioavailability of the drug which in turn improves patient compliance [4]. The natural or synthetic macromolecules are present that adhere to the mucus layer by interacting with the glycoproteins in the mucus, not by covalent bonding, but by weak ionic interactions such as hydrogen bonding and van der Waal's forces $[1,5]$. Nowadays, efforts are being made to increase the mucoadhesive properties of the polymer such as improved resistance to enzymes, increased cohesive force and facilitated paracellular diffusion of the drug, increased bioavailability, and increased patient compliance $[2,6]$. For this purpose, the new generation of mucoadhesive polymers developed by integrating sulfhydryl groups on the backbone of the polymer resulted in thiolated polymers, also called as thiomers $[6,7]$. This structural modification in the polymer has led to the improvement in the mucoadhesive properties of the polymers by 2 - to 140 -fold [8]. Polymers modified are

(i) alginate,

(ii) chitosan [2],

(iii) polycarbophil [9],

(iv) polyacrylic acid [1],

(v) xyloglucan [5],

(vi) carboxy methyl cellulose [10],

(vii) polyaspartamide $[11,12]$,

(viii) hydroxyethyl cellulose [13]. 
TABLE 1: Examples of cationic and anionic polymers.

\begin{tabular}{ll}
\hline Cationic thiomers & Anionic thiomers \\
\hline & Polyacrylic acid cystamine conjugate \\
& Sodium carboxy methyl starch (CMS) \\
Hydroxyethyl cellulose & Carboxy methyl guar gum (CMG) \\
& Carboxymethyl cellulose \\
& Sodium alginate \\
\hline Chitosan & \\
\hline
\end{tabular}

\section{Types of Thiomers}

Followings are the types of thiomers:

(i) Cationic thiomers.

(ii) Anionic thiomers.

2.1. Cationic Thiomer. Basically these are the thiomers based on chitosan, prepared by immobilizing thiol group on 2amino position of the glucosamine, present in the polymer chain. Examples include chitosan cysteine, chitosanthioglycolic acid, chitosan-thio butyl amidine, and so on (Table 1) [2].

2.1.1. Chitosan. Chitosan, a natural mucoadhesive polysaccharide polymer, obtained from deacetylation of chitin present in the crustacean shell, is chemically named as [poly $\{(1,4)$-2-amino-2-deoxy-d-glucopyranose $\}$ ]. It consists of two subunits $\mathrm{N}$-acetylglucosamine and glucosamine [14]. Chitosan has the mucoadhesive and permeation enhancing properties due to interaction of the amino group with the anionic structures in the mucus or epithelial layer due to its polycationic nature [15]. The primary amino group present in glucosamine is easily protonated in acidic media which increases its solubility, while it remains stable in basic and neutral media $[2,16]$.

Modified generation of chitosan is prepared with more pronounced mucoadhesive properties by incorporating thiol group on the backbone of the polymer that is creating the thiolated chitosan [17]. Thiolated chitosan is with improved mucoadhesive properties, enhanced permeation, and in situ gelling properties such as chitosan thio butyl amidine conjugate, chitosan cysteine conjugate, and chitosan thioglycolic acid conjugate $[2,18]$.

2.1.2. Increased Mucoadhesive Property of Chitosan. Thiolated chitosan is the modified polymer having ability to bind with the mucin through covalent bonding rather than weak ionic interactions such as hydrogen bonding or van der Waal's forces as simple chitosan. Sulphur present in the thiolated chitosan makes disulfide bonding with the cysteine rich domain of the mucin thus increasing time to stay in body which in turn increases bioavailability and also may help to reduce dosing frequency which ultimately increases patient compliance [2].

2.1.3. Mucin. Mucin is a glycoprotein consisting of protein core with branched oligosaccharides over $63 \%$ of its length and makes $5 \%$ of total mucus. It accounts for gel like properties of the mucus. Cysteine present in the mucin glycoprotein accounts for $1.5 \%$ of the amino acid in the small intestine that is about $9 \mu \mathrm{mol} \mathrm{SH} / \mathrm{g}$ of mucus [2].

2.1.4. Preparation of Thiolated Chitosan. Thiolated chitosan is prepared by introducing the sulfhydryl bearing moiety on the 2-amino position of the glucosamine subunit. This is done by reacting carboxylic group of the moiety such as cysteine or thioglycolic acid with the amino group in the polymer back bone leading to formation of the amide bond or amidine bond, in the presence of 1-ethyldiaminopropyl carbodiimides (EDAC) or thiobutyric acid, respectively, as coupling agent. Thiol group may undergo oxidation so synthesis must be done under inert conditions or at $\mathrm{pH}<5$, as at this $\mathrm{pH}$ the concentration of the thiol anions is low so disulfide formation seldom occurs $[2,4,19]$.

The schemes of reactions involved in the formation of thiolated chitosan are shown in Figure 1. It shows two schemes indicating one step process and two-step process. Single step processing of chitosan to achieve thiolated chitosan shows reaction medium consisting of water. The thiolation takes place in the presence of carbodiimide (EDAC) and NHS (Nhydroxysuccinimide). While the second process involves the two steps, first step involves DMF (N,N-dimethylformamide) as a reaction medium to avoid hydrolysis of the ester formed thereby in the reaction. This scheme is shown in Figure 1 [16].

2.1.5. Degree of Effective Thiol Immobilization. Studies have shown that immobilization of $25-250 \mu \mathrm{mol}$ thiol groups per gram of the chitosan is effective in improving the mucoadhesive properties [20] and permeability of the chitosan polymer. If the thiol groups are reduced their immobilization can be determined by Ellman's reagent, while if they are oxidized then by borohydride. Alternatively iodine titration method can be used [4].

2.1.6. Effect on Mucoadhesion. Mucoadhesive properties of the chitosan are enhanced by 5-10-fold in case of thioglycolic acid conjugate and 10-20-fold in case of chitosan-4thiobutylamidine conjugate $[2,21]$. The main perspective of thiolation is to increase the mucoadhesion. Mucoadhesion of different polymers increase by many folds with the addition of thiol group (Table 2) [22, 23].

2.1.7. Mechanism of Mucoadhesion of Thiomers [2]. Mucin is present all over the mucus membrane which has cysteine rich domains. The thiol group interact with these cysteines and lead to formation of the disulfide bonds either by oxidation of the thiol groups or by thiol/disulfide exchange reaction (Figure 2).

The extent to which the mucoadhesion takes place depends upon the $\mathrm{pKa}$ of the thiol group, $\mathrm{pH}$ of thiolated chitosan, and $\mathrm{pH}$ of the surrounding medium. The disulfide bonds thus formed are not influenced by the ionic strength and $\mathrm{pH}$ condition.

2.1.8. In Situ Gelling of Chitosan [24]. Ability of chitosan to crosslink adds more to its mucoadhesive efficiency. For 


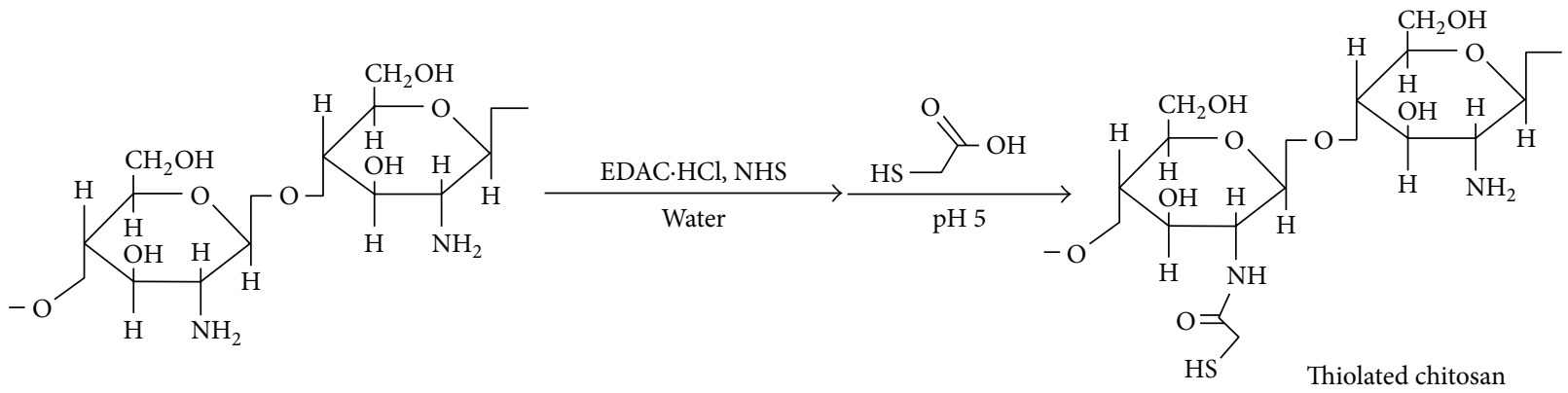

Chitosan

(-SH degree was about $231.51 \mu \mathrm{mol} / \mathrm{g}$ )

One-step reaction<smiles>CCN=C(CCCC[NH+](C)Cl)N=CC[NH+](C)Cl</smiles>

TGA

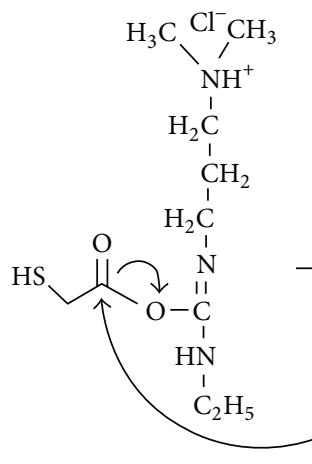

Unstable reactive

o-acylisourea ester

NHS

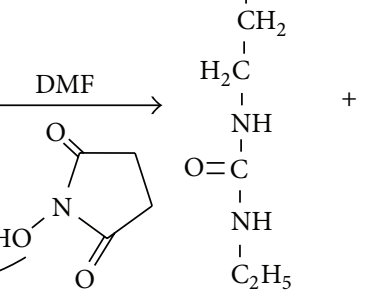<smiles>C[NH+](C)C</smiles>

$\mathrm{H}_{2} \mathrm{C}$

$\stackrel{\mathrm{C}}{\mathrm{C}} \mathrm{H}_{2}$<smiles>[O]C1CCCC1</smiles><smiles>O=C(CS)ON1CCCC1=O</smiles>

Reactive NHS-ester

The first step

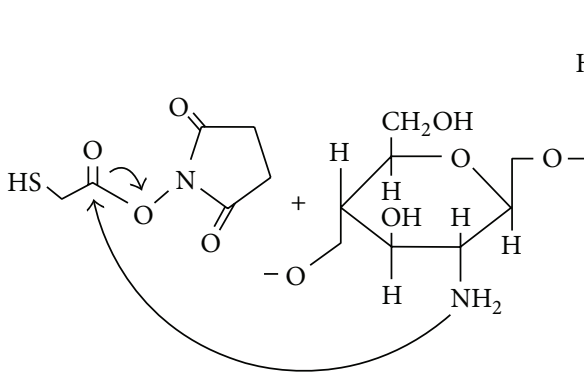

Reactive NHS-ester
Chitosan

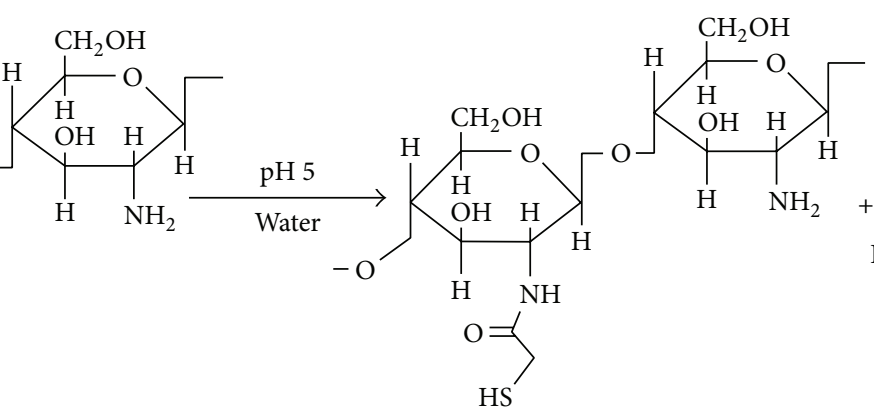

Thiolated chitosan (-SH degree was about $1411.01 \mu \mathrm{mol} / \mathrm{g}$ )<smiles>O=C1CCC(=O)N1O</smiles>

NHS

The second step

FIgURE 1: Process for thiolation of chitosan.

the chitosan, after forming surface interaction, it starts to form bonds with itself leading to more strong adhesion. The in situ gelling property of chitosan has been of great value for nasal, vaginal, and other preparations [2].

Thiolated chitosan has shown more pronounced gelling property in comparison to unmodified chitosan. The thiol groups interact with each other leading to formation of more strong gel. This occurs due to oxidation of the thiol group at physiological $\mathrm{pH}$. At $\mathrm{pH} 5.5$, the sol gel transition of the thiolated chitosan occurs after $2 \mathrm{~h}$. When the thiolated chitosan is rheologically observed for the gelling property, it has shown significant decline in the thiol group concentration showing formation of disulfide bond formation. Also, thiolated chitosan shows more elasticity in comparison. The in situ gel forming property of thiolated chitosan is important in case of liquid or semisolid preparation for nasal, vaginal, 
TABLE 2: Thiomers with their effect on mucoadhesion [11].

\begin{tabular}{ll}
\hline Thiomer & Increase in mucoadhesive strength \\
\hline Chitosan iminothiolane & 250 times \\
Polyacrylic acid-cysteine & 100 times \\
Polyacrylic acid-homocysteine & Approx. 20 times [21] \\
Chitosan thioglycolic acid & 10 times \\
Chitosan-thioethylamidine & 9 times \\
Alginate cysteine & 4 times \\
Poly methacrylic acid-cysteine & Improved cohesiveness and mucoadhesion [42, 43] \\
Sodium carboxy methyl cellulose-cysteine & Increased mucoadhesion \\
Gellan gum-2-iminothiolane & Increased mucoadhesion [44] \\
Carbopol 980 & Increased mucoadhesion [45] \\
Hyaluronicacid-L-cysteine & Increased mucoadhesion [46] \\
Carboxymethyl hyaluronic acid & Improved cohesiveness and mucoadhesion [47] \\
Poly(ethylene glycol)-glutathione & Improved drug delivery [48, 49] \\
Sodium alginate & Improved drug delivery [27] \\
\hline
\end{tabular}

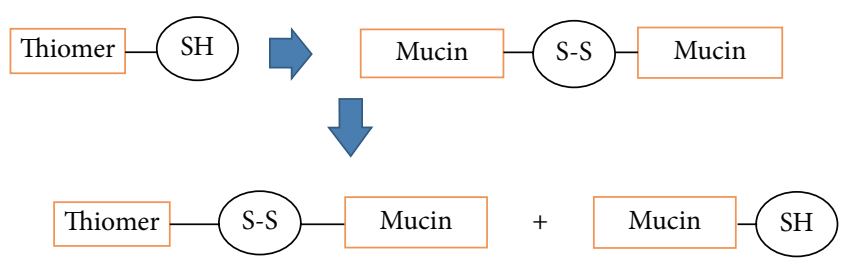

FIGURE 2: Mechanism of mucoadhesion of thiomers.

or ocular use, where thin layer of solution is applied which forms gel layer on mucus and thereby increases the contact time and thus bioavailability of the drug in the body [2].

2.1.9. Effect on Permeation. Thiolation of chitosan enhances its permeation effect many folds as compared to the simple chitosan. It can be assayed by using Ussing chamber, through the fresh intestinal mucosa. The studies have shown increase in the uptake of fluorescent bacitracin by 1.6-fold using chitosan cysteine, while, for cysteine-TBA it has shown increase of cationic marker rhodamine 123 uptake by 3 -fold as compared to unmodified chitosan [2].

2.1.10. Mechanism of Permeation Enhancement. Permeation enhancement effect of the thiolated chitosan can be explained by its effect on protein tyrosine phosphatase, the enzyme that is responsible for dephosphorylation of the transmembrane occludin tyrosine, the protein responsible for opening and closing of these tight junctions between cells. Thiolated chitosan inhibits this dephosphorylation to increase the permeation for drug, thus increasing bioavailability [2].

\subsubsection{Thiolated Chitosan in Different Dosage Forms}

Microparticles. Microparticulate dosage forms due to their small size have the ability to increase the bioavailability due to their increased time to stay in the body. Now when thiolated chitosan with improved mucoadhesive property and being more resistant to degradation is incorporated, the dosage form with more pronounced properties will be obtained [2]. Microparticles of thiolated polycarbophil were also prepared and shown to have 3 -fold mucoadhesion as compared to unmodified polycarbophil [25].

Tablets. $40 \%$ of all formulations are tablets. Thiolated chitosan can be incorporated for the prolonged release effect to the dosage form due to its in situ gelling properties. If only drug is mixed with polymer and compressed into tablet, it can still show zero order release for several hours. It shows hydration and diffusion account for the release rate [2].

Solutions. Thiolated chitosan seems to be unstable when in solution form due to its gelling property and disulphide bond formation within the polymer. This polymer used in solution formulations for eye can be effective in the most common eye problem, dry eye, the condition in which mucous layer of eye acting as a surfactant becomes defective, and there it can be used to form protective layer to cope with this condition [2].

Gels. Thiolated chitosan gel formulation seems to be very effective as compared to simple chitosan gels due to the thiolated back bone which enhance its mucoadhesive properties $[2]$.

2.1.12. Hydroxyethyl Cellulose. Hydroxyethyl cellulosecysteamine is synthesized by open ring oxidative amination of the hydroxyethyl cellulose to incorporate thiol groups on the polymer chain. The modified polymer thus obtained has 3-fold more viscosity in comparison to the unmodified hydroxyethyl cellulose. Also the swelling properties and the mucoadhesion are markedly improved $[16,26]$.

2.2. Anionic Thiomer. Carboxylic acid group is present as the substrate in anionic thiomers. Presence of carboxylic groups makes the immobilization of the thiol groups to these polymers more efficient by amide bond formation in the presence of carbodiimides. Examples include 


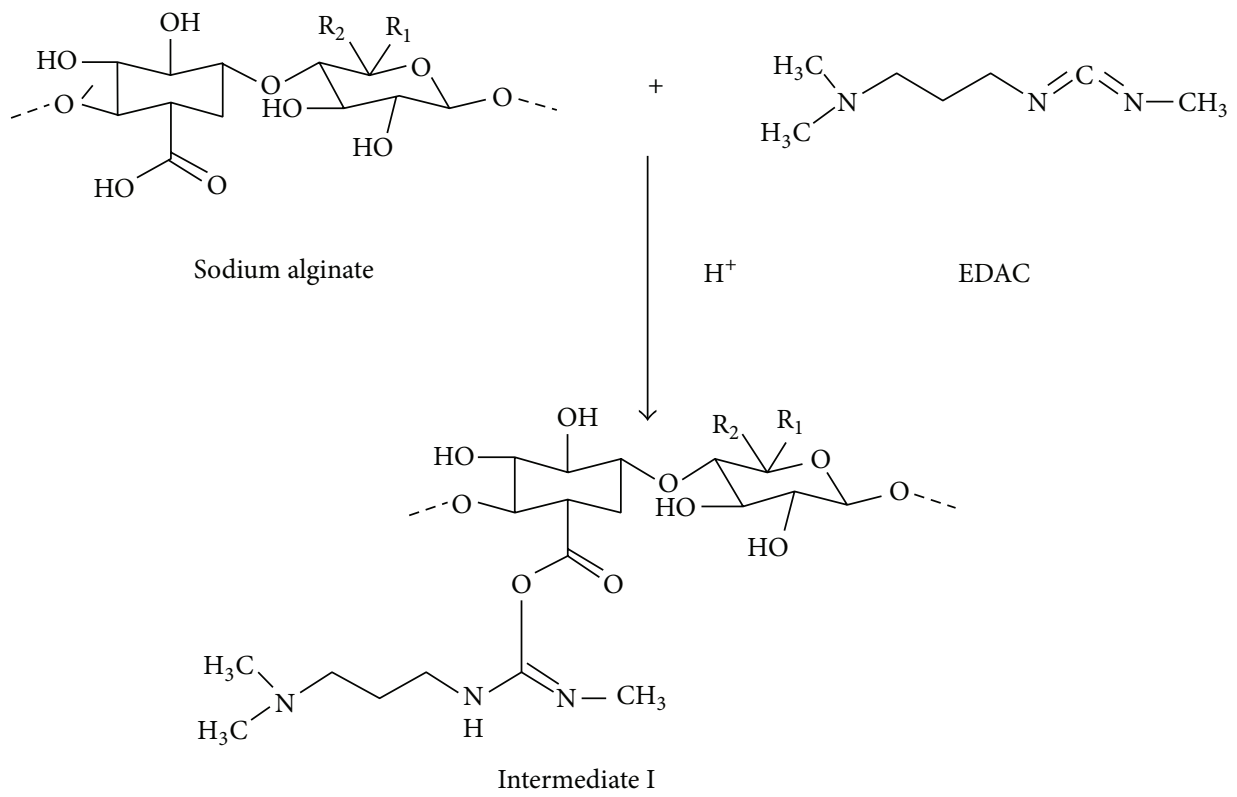

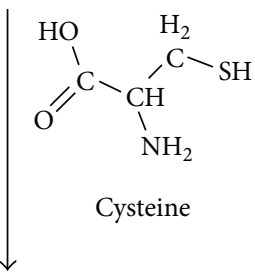

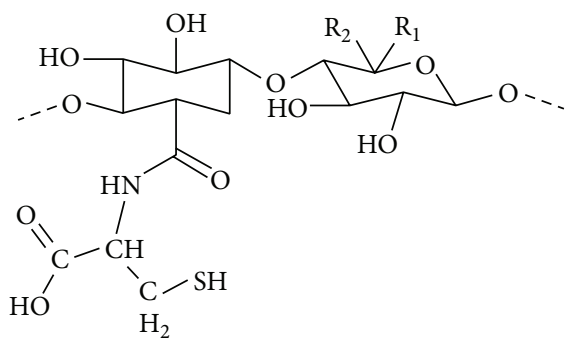<smiles>CNC(=O)NCCCN(C)C</smiles>

Urea derivative of EDAC

Sodium alginate cysteine conjugate

FIgURE 3: Process for thiolation of sodium alginate.

polyacrylic acid-cysteamine, CMC-cysteine, and alginatecysteine (Table 1) [11].

2.2.1. Sodium Alginate Cysteine Conjugate. Sodium alginate is thiolated for surplus effects. In the presence of carbodiimide thiolation takes place (Figure 3 ) and the thiomer thus produced is studied and shown to have $50 \%$ more viscosity in comparison to the simple sodium alginate solution at $37^{\circ} \mathrm{C}$. The swelling index is also improved [27]. Tablets thus prepared by using this modified polymer have shown to be more stable and resident on mucosa [28].

2.2.2. Polyacrylic Acid Cystamine Conjugate. Polyacrylic acid cystamine conjugates are prepared in the presence of carbodiimides by hydrolyzing polyacrylic acid polymer in demineralized water. Hydrolyzed polymer shows up its carboxylic acid group for S-S conjugation [29].

Polyacrylic acid homocysteine conjugates prepared in the presence of carbodimide have shown to contain sulfur $930 \mu \mathrm{mol} \pm 83$ per gram of thiomer. Polyacrylic acid homocysteine has shown to have improved mucoadhesive characteristics as compared to unmodified polymer.

2.2.3. Sodium Carboxy Methyl Starch (CMS) and Carboxy Methyl Guar Gum (CMG) [30]. Thiomers of CMS and CMG are prepared in the presence of carbodimide by coupling reaction. Thiol groups in the form of cysteine are conjugated. Thereafter the mucoadhesive properties of both the polymers are studied which showed CMS to be less mucoadhesive than CMG. On the other hand the swellability of CMG is not 
affected by cysteine immobilization. On the whole CMG has proven to be better candidate as release of drug from the matrix tablet is 1.5 -fold compared to CMS.

2.2.4. Thiolated Xyloglucan. Xyloglucans are important glycans that are cellulose microfibers obtained from the seeds of the dicots. It is thiolated and its thiolation can be determined by Ellman's reagent while mucoadhesive extent can be determined by in situ gelling of the modified polymer [5].

\section{Properties of Thiomers}

Hydrophilic properties of thiomers due to the thiol group make the polyacrylic acid and chitosan more mucoadhesive [11]. Other properties include cohesive properties, mucoadhesive properties, enzyme inhibition, and permeation enhancement [7].

\section{Characterization of Thiomers [29]}

Thiomers are tested and characterized for their stability and effectiveness by various methods such as.

4.1. Determination of Thiol Group Content [12]. The thiol group content on the polymer was determined by iodometry according to given procedure. The amount of thiol group tells the degree of thiolation. Hydrating with demineralized water thiomer was taken in the iodine flask. The $\mathrm{pH}$ of the solution was adjusted by adding $1 \mathrm{M} \mathrm{HCl}$ and then standard solution of $0.1 \mathrm{~N}$ iodine was added and shook for 30 minutes. Excessive iodine was titrated with $0.1 \mathrm{~N}$ sodium thiosulphate solution. Sarch is used as an indicator.

Same procedure mentioned above is used for estimation of blank without thiomer. The degree of thiolation is determined by following formula:

\%Thiol group content

$$
=\frac{[(\text { Blank }- \text { Proper }) \times 0.1 \times 0.066 \times 100]}{0.1}
$$

$\times$ weight of thiomer.

4.2. Disulfide Bond Formation [12]. $200 \mathrm{mg}$ of thiomer is hydrated with iodine in a flask and $\mathrm{pH}$ was adjusted between 2 and 3 using $1 \mathrm{M} \mathrm{HCl}$. Then $0.6 \mathrm{~mL}$ of $3 \%$ solution of sodium borohydride was added to the polymer and shaken for $15 \mathrm{~min}$ to hydrate all the disulfide bonds to free thiol groups. After this the mixture is neutralized by addition of $0.5 \mathrm{~mL}$ of $1 \mathrm{M}$ $\mathrm{HCl}$. The thiomer before reduction and after reduction of thiol groups is subtracted to estimate disulfide content [31]:

$\%$ Disulfide group content

$$
=\frac{\left[\left(B_{1}-B_{2}\right) \times 0.1 \times 0.066 \times 100\right]}{0.1}
$$

$\times$ weight of thiomer,

where, $B_{1}=$ Blank, burette reading after reduction; $B_{2}=$ Blank, burette reading before reduction.
4.3. Swelling Behavior [12]. Thiomers prepared at different $\mathrm{pH}$ are compared. $30 \mathrm{mg}$ of thiomer was compressed into a disc of $5 \mathrm{~mm}$ diameter and dipped in demineralized water at $20^{\circ} \mathrm{C}$ by placing at permeable bottom tubes.

4.4. Viscosity [12]. The viscometer is used to measure the viscosity of thiomers. $2 \%$ solution is prepared and temperature conditions of $25 \pm 1$ were used at $20 \mathrm{rpm}$.

4.5. Extent of Mucoadhesion: In Vivo. The freshly excised intestinal mucosa of the sheep was taken to study the extent to which thiomer binds to the mucosa. The mucosa was clamped upside down and the exposed area was about $0.785 \mathrm{~cm}^{2}$. Equal amounts of all formulations are used to equilibrate each part. Every sample mucosa was exposed for 2 minutes with formulation to ensure the intimate contact between the two. The weights are used to detach the mucosa clamped in the pan to another; the weights thus used to detach mucosa are expressed as detachment stress which will be used to estimate in vivo mucoadhesion of thiomer in dynes $/ \mathrm{cm}^{2}$. Consider

$$
\text { detachment stress }\left(\text { dynes } / \mathrm{cm}^{2}\right)=m * \frac{g}{A},
$$

where $m$ is mass, $g$ is acceleration due to gravity, and $A$ is area of tissue exposed.

\section{Routes of Drug Administration Using Thiomers as a Carrier of the Drugs}

There are various routes of drug administration that can be used to deliver the drug using thiomers as carrier. They have various applications using these routes of administration. The thiomer preparations are available for oral, nasal, ocular, buccal delivery of the drug. In literature, it is studied that thiomers can be used to deliver the drug through transmucosal route, gastrointestinal route, buccal route, oral route, nasal route, ophthalmic route, and vaginal route of drug delivery. Different routes of drug administration along with the drugs that can be used through these routes are discussed as follows.

5.1. Oral Delivery of Drug. Oral route of drug administration is considered as safe and effective for various drugs. Effects of various drugs can be enhanced when they are administered through oral route using thiolated polymers such as increased stay of drug in intestine and prolonged contact, enhanced permeation, and enzyme inhibition [32]. Bioavailability of poorly bioavailable drugs like calcitonin, insulin, and low molecular weight heparin can be increased using thiomers based formulations. There are various applications of thiolated polymers in enhancement of bioavailability of poorly bioavailable drugs through oral route for example.

Calcitonin: calcitonin bioavailability is improved by conjugating it with the chitosan in modified form as compared to simple one due to the enzyme inhibiting and permeation enhancement effect [33]. It led to $5 \%$ more decrease in the calcium level [9]. 
Insulin: bioavailability and effect of insulin can be enhanced using thiolated polymer as the carrier in the form of matrix tablets. Thiolated polycarbophil using cysteine was used to carry the insulin in the form of tablets. This formulation showed $36 \%$ more decrease in the blood sugar level comparatively [9].

Low molecular weight heparin: low molecular weight heparin is administered subcutaneously which is painful and noncompliant; thus there is a need for the development of the oral drug delivery system which will provide improved bioavailability. Thiolated poly acrylic acid was used as a carrier to deliver the LMW heparin orally. It was observed that formulating low molecular weight heparin with thiolated polymers has shown to provide relatively improved bioavailability $(5.8 \pm 1.4 \%)$ [9]. LMW heparin has shown absolute bioavailability of $\geq 20 \%$ in rats [34].

5.2. Transmucosal Delivery of Drug. Thiomers have the potential to deliver the drugs through transmucosal routes comparatively with better effects. Thiomers have the ability to provide the control drug release. In a study thiolated chitosan was prepared using thio butyl amidine as a conjugating agent. Chitosan thio-butyl-amidine microspheres were prepared to evaluate the controlled delivery of the active pharmaceutical ingredients. The results have showed the controlled release of drug through these microspheres. Thus thiomers can be effectively used for the less absorbed drugs to be delivered through mucosa $[35,36]$. It is also concluded that the thiolated chitosan can be a good candidate for the formulation as beads for the controlled release of the drug [17].

5.3. Gastrointestinal Delivery of Drug. The recent advance in the polymer technology has led to improving in the drug delivery. The hydrophilic macromolecular drugs now can be effectively delivered using thiomers due to increased contact time by mucoadhesion and also due to enzyme inhibiting properties of thiomers as in case of GI peptidase [37].

Peptides: peptide delivery can be enhanced by incorporating it with thiomers. The oxidation of peptide is reduced and also the permeation of drug through the gut is increased due to increased stay of the formulation in the body. Thiolated chitosan can enhance the stability and dissolution profile of the orally delivered peptides. One of the formulations for the oral delivery of peptides is by using the thiolated chitosan and chitosan was thiolated as chitosan-thiol-butylamidine. It is compressed with tablets to deliver in gut. These tablets are coated with triglycerides to prevent adhesion in oral cavity or esophagus. In stated study enhanced bioavailability as well as stability was observed [30].

5.4. Buccal Delivery of Drug. Mucoadhesive buccal formulations are getting more and more intention. Thiolated polymers can be effectively used to enhance the buccal delivery of drugs especially the drugs with problems through transGIT delivery of the drugs. L-cysteine conjugates were used to prepare the matrix tablets and there after the unmodified and modified polymers used in preparation are further studies for effect on mucoadhesion. Thiomers thus prove to be an effective excipient for buccal delivery of the drugs [38].
5.5. Nasal Delivery of Drug. Thiomers can also be effective carrier for the delivery of drug through nasal route. Enhanced mucoadhesion and permeation make the thiomers effective tool for the delivery of drug through this route. Nasal route has been shown to be effective for delivery of drug using thiomers as permeation enhancement effect is more prominent. Various thiolated polymers including chitosan, sodium alginates, and polycarbophil can be used for the nasal delivery of the drug. Thiolated polycarbophil was used to study the leu-enkaphlin delivery on the bovine mucosal cells. The results showed delivering the drug through the thiomer by sustained release and the degradation of drug is lowered. Also there was increase in drug uptake by nasal mucosa by 80 -fold. Thus thiolated polycarbophil can prove to be a promising tool for nasal delivery drug [35].

Human growth hormone (hGH): thiolated polycarbophil and glutathione were used for the delivery of hGH through nasal route in the form of hydrogel. It was observed that this formulation has effectively improved the plasma level of the drug [9].

5.6. Ophthalmic Delivery of Drug. Ophthalmic drug delivery is an effective route of administration but it also has various drawbacks and the important one is decreased retention time of the drug. Various thiomers including thiolated chitosan, thiolated sodium alginate, thiolated poly ethylene glycol, and polycarbophil have the potential to deliver the drug effectively with increased mucoadhesion. A thiomer of nonionic surfactant; that is, cysteine-PEG was used to formulate the nanoparticulate preparation of the cyclosporine to deliver through the ophthalmic route and was shown to remain in the ocular cul-de-sac for about $6 \mathrm{~h}$. Thus it has shown to increase the stay and also the concentration of drug in ocular region as compared to normal lipid carriers [39]. Similarly ocular inserts of using thiomers are carrier like thiolated polyacrylic acid that can also be formulated to improve the bioavailability as well as effectiveness of the drugs [35]. Various studies showed that plasmid DNA encoded with green fluorescence protein can be delivered with improved effects in a study; it was nanocomplexed with modified and unmodified chitosan to compare the effects of both carriers. Thereafter the transfection efficiency of thiolated chitosan and the sustained action produced by the thiolated chitosan was found greater comparatively $[35,40]$.

5.7. Vaginal Delivery of Drug. Delivery of drug through intravaginal route using thiomers offers various advantages like increased in situ gelling, controlled release, mucoadhesion, and enzyme inhibition thereby increasing the concentration of drug delivered. Thiomeric formulation to be administered through vaginal route can be in the form of tablets, capsules, gels, liquids, and so forth and once administered they remain there to deliver drug at a controlled release rate for even weeks [35].

In a study thiolated carbopol was used for vaginal delivery of LH-RH to observe the effect of thiomer on aminopeptidase $\mathrm{N}$. it was observed that enzyme inhibition is greatly linked with thiol concentration. Increase in the concentration of thiol group improves the enzyme inhibition [41]. 


\section{Conclusion}

The improvement in the mucoadhesive properties of the polymers by incorporation of the thiol groups provides us with the polymers and ultimately the dosage form with multiple additive properties such as taste masking, improved permeation, prolonged release, reduced irritation, and patient compliance. Different polymers that are modified by addition of thiol groups are used in different preparations for different routes accordingly. By using these modified polymers the plasma drug levels are increased.

\section{Conflict of Interests}

The authors declare that there is no conflict of interests regarding the publication of this paper.

\section{References}

[1] S. Roy, K. Pal, A. Anis, K. Pramanik, and B. Prabhakar, "Polymers in mucoadhesive drug-delivery systems: a brief note," Designed Monomers and Polymers, vol. 12, no. 6, pp. 483-495, 2009.

[2] K. Deepak, M. S. Kumar, and N. Mahadevan, “Thiolated chitosan: modified advanced generation of mucoadhesive polymers," International Journal of Recent Advance in Pharmaceutical Research, vol. 2, no. 3, pp. 31-41, 2012.

[3] G. Shahnaz, G. Perera, D. Sakloetsakun, D. Rahmat, and A. Bernkop-Schnürch, "Synthesis, characterization, mucoadhesion and biocompatibility of thiolated carboxymethyl dextrancysteine conjugate," Journal of Controlled Release, vol. 144, no. 1, pp. 32-38, 2010.

[4] A. Anitha, N. Deepa, K. P. Chennazhi, S. V. Nair, H. Tamura, and R. Jayakumar, "Development of mucoadhesive thiolated chitosan nanoparticles for biomedical applications," Carbohydrate Polymers, vol. 83, no. 1, pp. 66-73, 2011.

[5] H. S. Mahajan, V. K. Tyagi, R. R. Patil, and S. B. Dusunge, "Thiolated xyloglucan: synthesis, characterization and evaluation as mucoadhesive in situ gelling agent," Carbohydrate Polymers, vol. 91, no. 2, pp. 618-625, 2013.

[6] K. Albrecht and A. Bernkop-Schnürch, "Thiomers: forms, functions and applications to nanomedicine," Nanomedicine, vol. 2, no. 1, pp. 41-50, 2007.

[7] G. Mythri, K. Kavitha, M. R. Kumar, and S. Jagadeesh Singh, "Novel mucoadhesive polymers-a review," Journal of Applied Pharmaceutical Science, vol. 1, no. 8, pp. 37-42, 2011.

[8] M. P. Wagh, O. U. Joshi, J. S. Patel, and V. R. Jain, "Thiomers: a new generation of mucoadhesive polymers," Research Journal of Pharmacy and Technology, vol. 2, no. 2, pp. 250-255, 2009.

[9] K. Kafedjiiski and L. Franzens, "Multifunctional polymeric excipients in no-invasive delivery of hydrophilic macromolecular drugs: the thiomer-technology," The Drug Delivery Companies Report Autumn/Winter 47, The Drug Delivery Companies, 2004.

[10] C. E. Kast and A. Bernkop-Schnürch, "Polymer-cysteamine conjugates: new mucoadhesive excipients for drug delivery?" International Journal of Pharmaceutics, vol. 234, no. 1-2, pp. 9199, 2002.

[11] M. B. Biličić, J. Filipović-Grčić, A. Martinac et al., "Synthesis and characterization of thiomers of polyaspartamide type,"
International Journal of Pharmaceutics, vol. 291, no. 1-2, pp. 211219, 2005.

[12] M. Barbarić, M. Kralj, M. Marjanović et al., "Synthesis and in vitro antitumor effect of diclofenac and fenoprofen thiolated and nonthiolated polyaspartamide-drug conjugates," European Journal of Medicinal Chemistry, vol. 42, no. 1, pp. 20-29, 2007.

[13] D. Rahmat, D. Sakloetsakun, G. Shahnaz, G. Perera, R. Kaindl, and A. Bernkop-Schnürch, "Design and synthesis of a novel cationic thiolated polymer," International Journal of Pharmaceutics, vol. 411, no. 1-2, pp. 10-17, 2011.

[14] R. Jayakumar, R. L. Reis, and J. F. Mano, "Synthesis and characterization of $\mathrm{pH}$-sensitive thiol-containing chitosan beads for controlled drug delivery applications," Drug Delivery, vol. 14, no. 1, pp. 9-17, 2007.

[15] S. Chopra, S. Mahdi, J. Kaur, Z. Iqbal, S. Talegaonkar, and F. J. Ahmad, "Advances and potential applications of chitosan derivatives as mucoadhesive biomaterials in modern drug delivery," Journal of Pharmacy and Pharmacology, vol. 58, no. 8, pp. 1021-1032, 2006.

[16] X. Zhu, M. Su, S. Tang et al., "Synthesis of thiolated chitosan and preparation nanoparticles with sodium alginate for ocular drug delivery," Molecular Vision, vol. 18, pp. 1973-1982, 2012.

[17] C. E. Kast, W. Frick, U. Losert, and A. Bernkop-Schnürch, "Chitosan-thioglycolic acid conjugate: a new scaffold material for tissue engineering?" International Journal of Pharmaceutics, vol. 256, no. 1-2, pp. 183-189, 2003.

[18] S. Hauptstein and A. Bernkop-Schnürch, "Thiomers and thiomer-based nanoparticles in protein and DNA drug delivery," Expert Opinion on Drug Delivery, vol. 9, no. 9, pp. 10691081, 2012.

[19] I. Bravo-Osuna, C. Vauthier, A. Farabollini, G. F. Palmieri, and G. Ponchel, "Mucoadhesion mechanism of chitosan and thiolated chitosan-poly (isobutyl cyanoacrylate) core-shell nanoparticles," Biomaterials, vol. 28, no. 13, pp. 2233-2243, 2007.

[20] K. Kafedjiiski, A. H. Krauland, M. H. Hoffer, and A. BernkopSchnürch, "Synthesis and in vitro evaluation of a novel thiolated chitosan," Biomaterials, vol. 26, no. 7, pp. 819-826, 2005.

[21] V. Grabovac, D. Guggi, and A. Bernkop-Schnürch, "Comparison of the mucoadhesive properties of various polymers," Advanced Drug Delivery Reviews, vol. 57, no. 11, pp. 1713-1723, 2005.

[22] C. E. Kast and A. Bernkop-Schnürch, "Thiolated polymersthiomers: development and in vitro evaluation of chitosanthioglycolic acid conjugates," Biomaterials, vol. 22, no. 17, pp. 2345-2352, 2001.

[23] X. Wang, J. Iqbal, D. Rahmat, and A. Bernkop-Schnürch, "Preactivated thiomers: permeation enhancing properties," International Journal of Pharmaceutics, vol. 438, no. 1-2, pp. 217-224, 2012.

[24] M. D. Hornof, C. E. Kast, and A. Bernkop-Schnürch, "In vitro evaluation of the viscoelastic properties of chitosanthioglycolic acid conjugates," European Journal of Pharmaceutics and Biopharmaceutics, vol. 55, no. 2, pp. 185-190, 2003.

[25] K. Albrecht, E. J. Zirm, T. F. Palmberger, W. Schlocker, and A. Bernkop-Schnürch, "Preparation of thiomer microparticles and in vitro evaluation of parameters influencing their mucoadhesive properties," Drug Development and Industrial Pharmacy, vol. 32, no. 10, pp. 1149-1157, 2006.

[26] D. Rahmat, C. Müller, J. Barthelmes, G. Shahnaz, R. Martien, and A. Bernkop-Schnürch, "Thiolated hydroxyethyl cellulose: design and in vitro evaluation of mucoadhesive and permeation 
enhancing nanoparticles," European Journal of Pharmaceutics and Biopharmaceutics, vol. 83, no. 2, pp. 149-155, 2013.

[27] M. Davidovich-Pinhas, O. Harari, and H. Bianco-Peled, "Evaluating the mucoadhesive properties of drug delivery systems based on hydrated thiolated alginate," Journal of Controlled Release, vol. 136, no. 1, pp. 38-44, 2009.

[28] M. Bhalekar, S. Sonawane, and S. Shimpi, "Synthesis and characterization of a cysteine xyloglucan conjugate as mucoadhesive polymer," Brazilian Journal of Pharmaceutical Sciences, vol. 49, no. 2, pp. 285-292, 2013.

[29] J. Iqbal, G. Shahnaz, S. Dünnhaupt, C. Müller, F. Hintzen, and A. Bernkop-Schnürch, "Preactivated thiomers as mucoadhesive polymers for drug delivery," Biomaterials, vol. 33, no. 5, pp. 1528-1535, 2012.

[30] N. Langoth, J. Kalbe, and A. Bernkop-Schnürch, "Development of buccal drug delivery systems based on a thiolated polymer," International Journal of Pharmaceutics, vol. 252, no. 1-2, pp. 141$148,2003$.

[31] V. M. Leitner, G. F. Walker, and A. Bernkop-Schnürch, "Thiolated polymers: evidence for the formation of disulphide bonds with mucus glycoproteins," European Journal of Pharmaceutics and Biopharmaceutics, vol. 56, no. 2, pp. 207-214, 2003.

[32] A. Bernkop-Schnürch, "Mucoadhesive systems in oral drug delivery," Drug Discovery Today: Technologies, vol. 2, no. 1, pp. 83-87, 2005.

[33] D. Guggi, C. E. Kast, and A. Bernkop-Schnürch, "In vivo evaluation of an oral salmon calcitonin-delivery system based on a thiolated chitosan carrier matrix," Pharmaceutical Research, vol. 20, no. 12, pp. 1989-1994, 2003.

[34] A. Bernkop-Schnürch, M. H. Hoffer, and K. Kafedjiiski, "Thiomers for oral delivery of hydrophilic macromolecular drugs," Expert Opinion on Drug Delivery, vol. 1, no. 1, pp. 8798, 2004.

[35] V. Ravi, C. Mahendra, M. V. Datta, D. V. Gowda, H. G. Shivakumar, and E. Bhargav, "Thiomers fresh drift of polymers \& their prospective in pharmaceuticals: a review," World Journal of Pharmacy and Pharmaceutical Sciences, vol. 3, no. 1, pp. 204220, 2013.

[36] M. Elhassan Imam and A. Bernkop-Schnürch, "Controlled drug delivery systems based on thiolated chitosan microspheres," Drug Development and Industrial Pharmacy, vol. 31, no. 6, pp. 557-565, 2005.

[37] A. Bernkop-Schnürch, A. H. Krauland, V. M. Leitner, and T. Palmberger, "Thiomers: potential excipients for non-invasive peptide delivery systems," European Journal of Pharmaceutics and Biopharmaceutics, vol. 58, no. 2, pp. 253-263, 2004.

[38] J. Shen, Y. Wang, Q. Ping, Y. Xiao, and X. Huang, "Mucoadhesive effect of thiolated PEG stearate and its modified NLC for ocular drug delivery," Journal of Controlled Release, vol. 137, no. 3, pp. 217-223, 2009.

[39] T. Schmitz, V. Grabovac, T. F. Palmberger, M. H. Hoffer, and A. Bernkop-Schnürch, "Synthesis and characterization of a chitosan-N-acetyl cysteine conjugate," International Journal of Pharmaceutics, vol. 347, no. 1-2, pp. 79-85, 2008.

[40] T. Schmitz, I. Bravo-Osuna, C. Vauthier, G. Ponchel, B. Loretz, and A. Bernkop-Schnürch, "Development and in vitro evaluation of a thiomer-based nanoparticulate gene delivery system," Biomaterials, vol. 28, no. 3, pp. 524-531, 2007.

[41] C. Valenta, M. Marschütz, C. Egyed, and A. Bernkop-Schnürch, "Evaluation of the inhibition effect of thiolated poly(acrylates) on vaginal membrane bound aminopeptidase $\mathrm{N}$ and release of the model drug LH-RH,' Journal of Pharmacy and Pharmacology, vol. 54, no. 5, pp. 603-610, 2002.

[42] E. Hrsic, H. Keul, and M. Möller, "Synthesis of thiol functionalized poly(meth)acrylates through enzymatic catalysis and a subsequent one pot reaction process," European Polymer Journal, vol. 48, no. 4, pp. 761-768, 2012.

[43] A. Kotal, S. Si, T. K. Paira, and T. K. Mandal, "Synthesis of semitelechelic POSS-polymethacrylate hybrids by thiolmediated controlled radical polymerization with unusual thermal behaviors," Journal of Polymer Science, Part A: Polymer Chemistry, vol. 46, no. 3, pp. 1111-1123, 2008.

[44] A. O. Andrade, M. E. Parente, and G. Ares, "Screening of mucoadhesive vaginal gel formulations," Brazilian Journal of Pharmaceutical Sciences, vol. 50, no. 4, pp. 931-941, 2014.

[45] D. Guggi, C. E. Kast, and A. Bernkop-Schnürch, "In vivo evaluation of an oral salmon calcitonin-delivery system based on a thiolated chitosan carrier matrix," Pharmaceutical Research, vol. 20, no. 12, pp. 1989-1994, 2003.

[46] K. Kafedjiiski, R. K. R. Jetti, F. Föger et al., "Synthesis and in vitro evaluation of thiolated hyaluronic acid for mucoadhesive drug delivery," International Journal of Pharmaceutics, vol. 343, no. 1-2, pp. 48-58, 2007.

[47] G. Yang, G. D. Prestwich, and B. K. Mann, "Thiolated carboxymethyl-hyaluronic-acid-based biomaterials enhance wound healing in rats, dogs, and horses," ISRN Veterinary Science, vol. 2011, Article ID 851593, 7 pages, 2011.

[48] A. Southan, E. Hoch, V. Schönhaar et al., "Side chain thiolfunctionalized poly(ethylene glycol) by post-polymerization modification of hydroxyl groups: synthesis, crosslinking and inkjet printing," Polymer Chemistry, vol. 5, no. 18, pp. 5350-5359, 2014.

[49] S. R. Williams, B. S. Lepene, C. D. Thatcher, and T. E. Long, "Synthesis and characterization of poly(ethylene glycol)glutathione conjugate self-assembled nanoparticles for antioxidant delivery," Biomacromolecules, vol. 10, no. 1, pp. 155-161, 2009. 

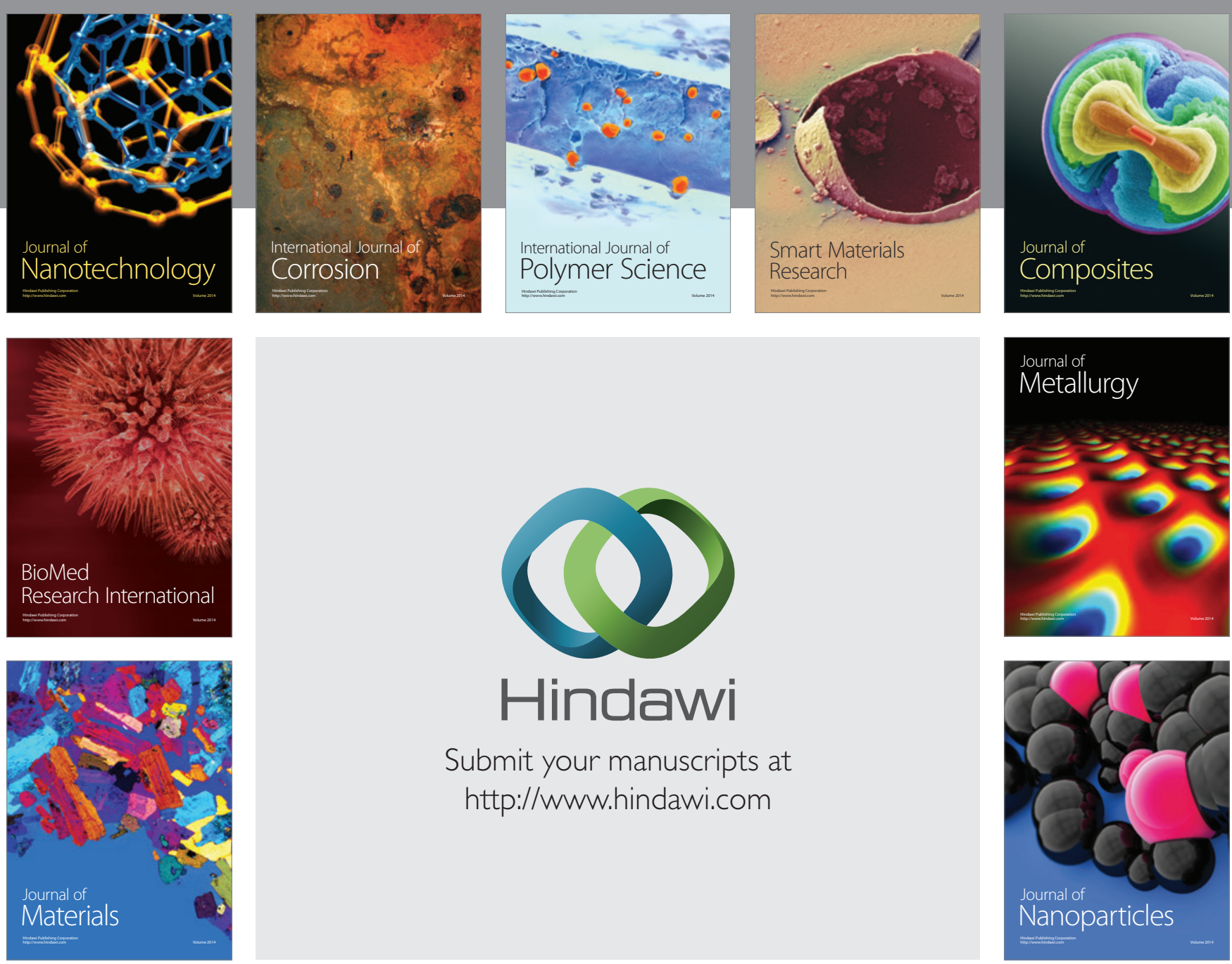

Submit your manuscripts at http://www.hindawi.com
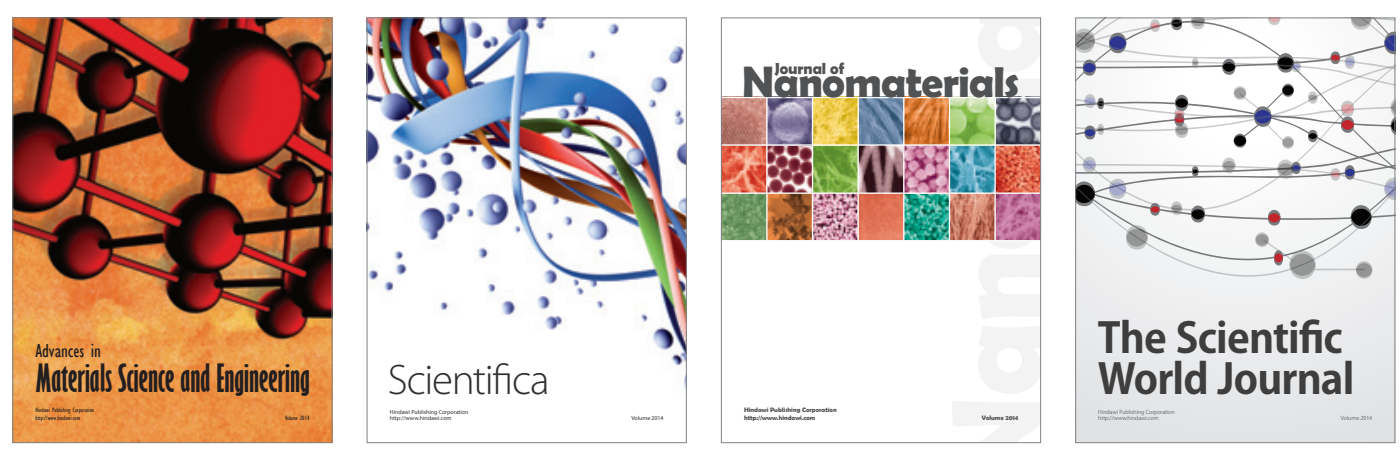

\section{The Scientific World Journal}
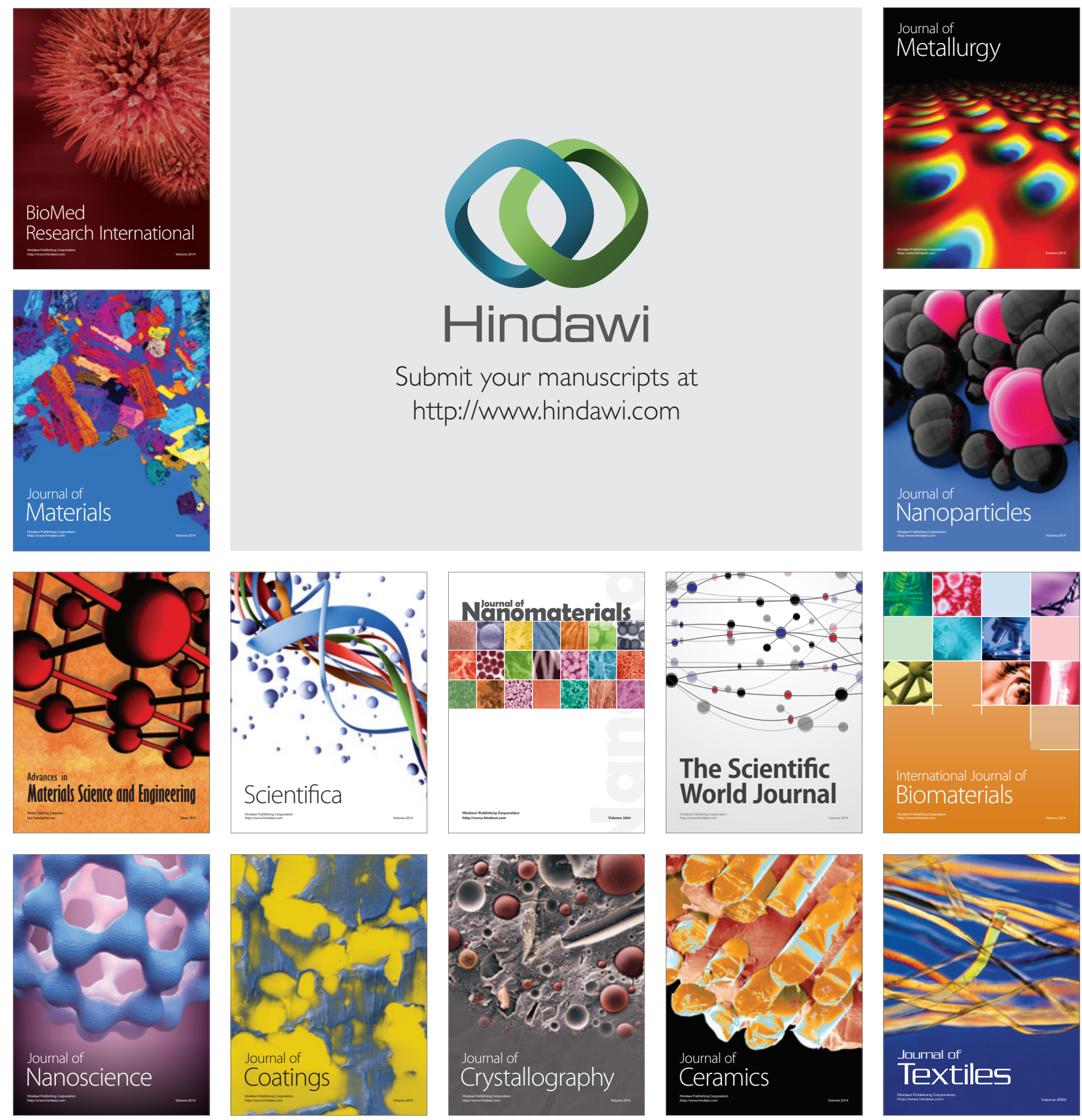